\title{
Multiyear tagging studies incorporating fishing effort data
}

\author{
John M. Hoenig, Nicholas J. Barrowman, William S. Hearn, and \\ Kenneth H. Pollock
}

\begin{abstract}
The Brownie models for multiyear tagging studies can be used to estimate age- and year-specific annual survival rates and tag recovery rates. The latter are composites of the exploitation rates and rates of tag reporting, tag shedding, and tag-induced mortality. It is possible to estimate the exploitation rates if the other components of the tag recovery rates can be quantified. Instantaneous rates of fishing and natural mortality can be estimated if information is available on the seasonal distribution of fishing effort. The estimated rates are only moderately dependent on the timing of the fishing; consequently, the relative effort data can be crude. Information on the timing of the catch over the course of the year can be used as a substitute for the effort data. Fishing mortality can also be assumed to be proportional to fishing effort over years; consequently, if fishing effort is known then the tag reporting rate, natural mortality rate, and a single catchability coefficient can be estimated (instead of natural mortality and a series of fishing mortalities). Although it is possible in theory to estimate both the tag reporting rate and the natural mortality rate with all of these models, in practice it appears necessary to obtain some additional data relating to tag reporting rate to obtain acceptable results. The additional data can come from a variable reward tagging study, a creel or port sampling survey, or from tagged animals that are secretly added to the fishers' catches.
\end{abstract}

Résumé : Les modèles de Brownie utilisés pour des études de marquage pluriannuelles peuvent servir à évaluer le taux de survie et le taux de récupération des marques pour des âges et des années donnés. Le taux de récupération des marques est un composite du taux d'exploitation et des taux de déclaration des marques, de pertes des marques et de la mortalité induite par les marques. On peut estimer les taux d'exploitation si les autres composantes du taux de récupération des marques peuvent être mesurées. Les taux instantanés de mortalité par pêche et de mortalité naturelle peuvent être évalués si on possède des données sur la répartition saisonnière de l'effort de pêche. Les taux estimés n'étant que modérément dépendants de la période de pêche, les données relatives sur l'effort peuvent donc être grossières. On peut remplacer les données sur l'effort par les données sur la période de capture au cours de l'année. On peut aussi supposer que la mortalité par pêche est proportionnelle à l'effort de pêche au cours des années; donc, si l'effort de pêche est connu, on peut alors évaluer le taux de déclaration des marques, le taux de mortalité naturelle et un coefficient unique de capturabilité (plutôt que la mortalité naturelle et une série de taux de mortalité par pêche). Tous ces modèles permettent d'évaluer théoriquement le taux de déclaration des marques et le taux de mortalité naturelle; mais, dans la pratique, il faudrait d'autres données sur le taux de déclaration des marques afin d'obtenir des résultats acceptables. Ces autres données peuvent provenir d'une étude de marquage avec récompense variable, d'une enquête sur la pêche sportive ou d'un échantillonnage au port, ou d'animaux marqués qui sont ajoutés à l'insu de pêcheurs dans leurs prises.

[Traduit par la Rédaction]

\section{Introduction}

Brownie et al. (1978, 1985) developed a series of models for multiyear tagging studies that allow one to estimate age- and year-specific survival rates and tag recovery rates. The latter are composite parameters involving tag retention, exploitation, tag-induced mortality, and tag reporting rates and, therefore,

Received January 22, 1997. Accepted June 6, 1997.

J13839

J.M. Hoenig ${ }^{1}$ and N.J. Barrowman. Department of Fisheries and Oceans, P.O. Box 5667, St. John's, NF A1C 5X1, Canada.

W.S. Hearn. CSIRO Division of Fisheries, Marine

Laboratories, G.P.O. Box 1538, Hobart, Tasmania 7001,

Australia.

K.H. Pollock. Department of Statistics, P.O. Box 8203, North

Carolina State University, Raleigh, NC 27695, U.S.A.

1 Author to whom all correspondence should be addressed. Present address: Virginia Institute of Marine Science, P.O. Box 1346, Gloucester Point, VA 23062, U.S.A. e-mail: hoenig@vims.edu are uninteresting parameters by themselves. The Brownie models generalize early work by Seber (1970) and Robson and Youngs (1971). Although Youngs and Robson (1975) applied their model to a study of lake trout (Salvelinus namaycush), it has only been recently that this class of models and its extensions have received much attention in fisheries research (Jagielo 1991; Larson et al. 1991; Pollock et al. 1991; Dorazio 1992; Schwarz et al. 1993).

Pollock et al. (1991) showed that, when additional information on the retention-survival rate and tag reporting rate is available, one can estimate the exploitation rate from the recovery rate parameter. If additional information is available on the seasonal distribution of fishing intensity, then it is possible to determine the instantaneous rates of fishing and natural mortality. Pollock et al. $(1991,1994)$ considered two special cases: constant fishing effort over the year and effort confined to a short pulse.

The Brownie models are extremely useful. However, a valid criticism is that they may not make use of all available information. For example, they do not take the magnitude of the fishing effort into consideration, and they do not include a 
Table 1. Expected and observed number of tag recoveries in a multiyear tagging study (model 1).

\begin{tabular}{lcccll}
\hline \multicolumn{5}{c}{$\begin{array}{c}\text { No. } \\
\text { Year }\end{array}$} & \multicolumn{5}{c}{ Year } \\
\cline { 3 - 6 } tagged & 1 & 2 & \multicolumn{1}{c}{3} & \multicolumn{1}{c}{4} \\
\hline \multicolumn{2}{l}{ Expected recoveries } \\
1 & $N_{1}$ & $N_{1} f_{1}$ & $N_{1} S_{1} f_{2}$ & $N_{1} S_{1} S_{2} f_{3}$ & $N_{1} S_{1} S_{2} S_{3} f_{4}$ \\
2 & $N_{2}$ & - & $N_{2} f_{2}$ & $N_{2} S_{2} f_{3}$ & $N_{2} S_{2} S_{3} f_{4}$ \\
3 & $N_{3}$ & - & - & $N_{3} f_{3}$ & $N_{3} S_{3} f_{4}$ \\
Observed recoveries & & & & \\
1 & $N_{1}$ & $r_{11}$ & $r_{12}$ & $r_{13}$ & $r_{14}$ \\
2 & $N_{2}$ & - & $r_{22}$ & $r_{23}$ & $r_{24}$ \\
3 & $N_{3}$ & - & - & $r_{33}$ & $r_{34}$ \\
\hline
\end{tabular}

Note: Symbols are as follows: $S_{j}$, survival rate in year $j ; f_{j}$, tag recovery rate in year $j ; r_{i j}$, number of tags recovered in year $j$ from animals tagged in year $i$.

functional relationship between survival rate and tag recovery rate. A second criticism is that the models are parameterized in terms of finite mortality rates instead of the instantaneous rates generally used by fisheries biologists. It would be valuable to be able to estimate the natural mortality rate. Furthermore, one might wish to impose restrictions on the component mortality rates, e.g., to specify constant natural mortality.

In this paper we concentrate on ways in which information on fishing effort may be used in the analysis of tagging data. We develop two approaches, one of which has a weak dependence on the effort data while the other has a strong dependence. For the former, the pattern of effort over the course of the year is used to partition the mortality into its fishing and natural components. In theory this can be done even if the tag reporting rate is unknown and estimated in the analysis. For the approach with the strong dependence on effort data we assume that fishing mortality is proportional to fishing effort over all years instead of just within a given year (i.e., that the catchability coefficient is the same for all years). There are two cases to consider. If reporting and retention-survival rates are known, then from two or more years of tag recovery data from a single release of tagged fish we can estimate natural mortality rate and the catchability coefficient (instead of estimating natural mortality and a series of individual annual fishing mortality rates). If reporting and retention-survival rates are not known, we can estimate the catchability coefficient, natural mortality rate, and the product of the tag reporting and retentionsurvival rates if tagged fish are released in at least 1 year and recaptures are made for at least 3 years. Alternatively, we can estimate these parameters from two or more years of recapture data when fish are tagged for each of 2 years.

\section{The Brownie models}

The structure of the Brownie models can be described in terms of the expected number of recaptures from each tagged cohort in each year (Table 1). We will assume that all animals tagged are fully recruited so that survival rate of the tagged animals does not change as the animals get older. Extension to the case of age-dependent survival rates is straightforward (Brownie et al. 1978, 1985). Suppose $N_{i}$ animals are tagged in year $i$ $(i=1,2, \ldots, I)$, and of these $r_{i j}$ are recovered in year $j(j=i$, $i+1, i+2, \ldots, J)$. The quantity $r_{i j}$ is a realization of the random variable $R_{i j}$ representing the expected number of recaptures; the expected value, $E\left[R_{i j}\right]$, is modelled as

$$
E\left[R_{i j}\right]= \begin{cases}N_{i} f_{j}, & i=j \\ N_{i} \prod_{h=i}^{j-1} S_{h} f_{j}, & j>i\end{cases}
$$

where $S_{h}$ is the annual survival rate for year $h$ (probability an animal alive at the start of year $h$ survives to the end of year $h$ ), and $f_{j}$ is the tag recovery rate for year $j$ (probability that a tagged animal alive at the start of year $j$ is caught during year $j$ and its tag is recovered). For convenience, let $E\left[R_{i j}\right]=N_{i} P_{i j}$. The recoveries for each cohort are modeled as a multinomial independent of all other cohorts. The likelihood $\Lambda$ is thus

$$
\Lambda \alpha \prod_{i=1}^{I}\left(\prod_{j=i}^{J} P_{i j}^{r_{i j}}\right) P_{i J^{*}} N_{i}-\Sigma_{j} r_{i j}
$$

where $P_{i j}$ is as above for $j=i, i+1, i+2, \ldots, J$ and

$$
P_{i J^{*}}=1-\sum_{j=i}^{J} P_{i j}
$$

$P_{i J^{*}}$ is the probability a tag from cohort $i$ is not recovered (by the end of year $J$ ). Various restrictions and generalizations are possible such as assuming survival is constant over time.

For three tagged cohorts and 4 years of recaptures (Table 1), it is possible to estimate the following parameters with the basic Brownie model: $f_{1}, f_{2}, f_{3}, S_{1}, S_{2}$, and the product $S_{3} f_{4}$. This can be seen by devising moment estimators from ratios of cell probabilities. For example,

$$
\begin{aligned}
& \hat{f}_{2}=\frac{r_{22}}{N_{2}} \\
& \hat{S}_{1}=\frac{N_{2} r_{12}}{N_{1} r_{22}} \text { or } \frac{N_{2} r_{13}}{N_{1} r_{23}} \text { or } \frac{N_{2} r_{14}}{N_{1} r_{24}} \\
& \hat{S}_{2}=\frac{N_{3} r_{23}}{N_{2} r_{33}} \text { or } \frac{N_{3} r_{24}}{N_{2} r_{34}} \\
& S_{3} \widehat{f_{4}}=\frac{r_{34}}{N_{2}}
\end{aligned}
$$

where the caret $\left({ }^{\wedge}\right)$ denotes an estimate. The moment estimation approach is useful for determining what parameters are estimable, and in certain cases it leads to maximum likelihood estimators. However, in general we obtain the maximum likelihood estimates by maximizing the likelihood function.

\section{Components of the tag recovery rate}

In this paper, we assume the tag recovery rate $f_{j}$ can be modeled as

(4) $f_{j}=\phi u_{j} \lambda_{j}$

where $\phi$ is the probability that an animal survives any initial tag shedding and initial tag-induced mortality, $u_{j}$ is the exploitation rate (probability that an animal present at the beginning of year $j$ is harvested during the year) and $\lambda_{j}$ is the tag reporting 
rate in year $j$ (probability that the tag will be reported given that the animal is harvested in year $j$ ). Often, $\lambda$ will be assumed constant over time. (Robson (1971) provided a $\chi^{2}$ test for constancy of $\lambda$ over time on the assumption that natural mortality is constant from year to year; however, the power of the test is not known.)

We refer to the product of the initial mortality caused by tagging and the short-term tag shedding rate as the retentionsurvival rate and denote this by $\phi$. The retention-survival rate can be estimated by holding newly tagged animals in cages or holding pens. We will also assume that the long-term or chronic tag shedding rate is minimal. This can be verified by double tagging experiments (e.g., Hearn et al. 1991; Barrowman and Myers 1996).

The tag reporting rate can be estimated by $(i)$ comparing the rate of return of tags with high-value rewards with the return rate of standard tags (Henny and Burnham 1976), (ii) estimating the number of tagged animals caught by a creel or port sampling survey and comparing this estimate with the number of tags voluntarily returned (Conroy 1985; Conroy et al. 1989; Pollock et al. 1991), and (iii) secretly placing tagged fish in fish traps or in the unprocessed catch on board fishing vessels and observing the fraction of tags returned (Costello and Allen 1968; Green et al. 1983). For completeness, we note that natural mortality and tag reporting rate can also be estimated effectively if fish are tagged before and after the fishing season each year (Hearn et al. 1998). However, as this involves a different type of study design, we do not consider this case further.

Thus, one can estimate the exploitation rate from the tag recovery rate, $f$, when external estimates of tag shedding, taginduced mortality rate, and reporting rate are available. Pollock et al. (1991) showed that, if there are different tag reporting rates for different groups of fishers, then cell probabilities must be specified separately for each group. In the Appendix, we show how to incorporate the different types of reporting data into the likelihood function.

Alternatively, we show below that it is possible to estimate the product $\phi \lambda$ from the tagging data, but it appears extremely difficult to achieve meaningful levels of precision without additional information on tag reporting rate.

\section{Instantaneous rates formulation}

Fishery scientists customarily work with instantaneous rates of fishing and natural mortality, $F$ and $M$, respectively. These can be derived from the exploitation rate and survival rate if the amount of fishing effort is known as a function of the time of year and the fishing mortality is assumed to be proportional to the amount of effort within periods of the year. Robson (1971) and Brownie (1974) developed a formulation for tagging models in terms of instantaneous rates, but they never published their results. Here, we present a more general formulation in which the seasonal pattern of effort can be of arbitrary form.

In year $j$, the exploitation rate, $u_{j}$, is a function of the fishing and natural mortality rates, which we can denote by $u_{j}\left(F_{j}, M_{j}\right)$ to emphasize the functional dependence. Suppose the fraction of the annual fishing effort in each period $k$ of year $j$ is denoted by $\varepsilon_{j k}$, for $k=1,2, \ldots, K$ (with $\Sigma_{k} \varepsilon_{j k}=1$ ). Let $\Delta t$ be the length of a period of the year expressed as a fraction of the year. Then (5a) $\quad u_{j}=u_{j}\left(F_{j}, M_{j}\right)=\sum_{k=1}^{K} a_{j k} b_{j k} c_{j k}$

where $a_{j k}$ is the fraction of the population surviving to the beginning of period $k\left(a_{j 1}=1\right)$

$$
a_{j k}=\exp \left(-M_{j}(k-1) \Delta t-F_{j} \sum_{h=0}^{k-1} \varepsilon_{j h}\right), \quad\left(\varepsilon_{j 0}=0\right)
$$

$b_{j k}$ is the fraction dying in period $k$

$$
b_{j k}=1-\exp \left(-M_{j} \Delta t-F_{j} \varepsilon_{j k}\right)
$$

and $c_{j k}$ is the fraction of the deaths in period $k$ due to fishing

$$
c_{j k}=\frac{F_{j} \varepsilon_{j k}}{F_{j} \varepsilon_{j k}+M_{j} \Delta t}
$$

while the annual survival rate is

(5b) $S_{j}=\exp \left(-M_{j}-F_{j}\right)$.

When fishing effort and hence fishing mortality are constant over the course of a year, eq. $5 a$ reduces to the familiar relationship (Ricker 1975):

$$
u_{j}\left(F_{j}, M_{j}\right)=\frac{F_{j}}{F_{j}+M_{j}}\left(1-\exp \left(-F_{j}-M_{j}\right)\right)
$$

and when fishing occurs instantaneously at the start of the year $K=1, \Delta t=0, \varepsilon_{j 1}=1$, and

$$
u_{j}\left(F_{j}\right)=1-\exp \left(-F_{j}\right)
$$

The expected values of the number of recoveries are given in Table 2 for the case where fishing occurs at the beginning of the year (Table 2A), throughout the year with constant intensity (Table 2B), and for the general case (Table 2C). Note that these expressions will need to be adjusted if the tagging episodes are not 1 year apart, e.g., if scheduling difficulties result in intervals between tagging ranging from 10 to 14 months.

\section{Models partitioning exploitation rate using seasonal effort data}

To estimate natural and fishing mortality rates, we substitute eq. $5 a$ into eq. 4 , and eq. $5 b$ and eq. 4 into the likelihood (eq. 2). The likelihood is maximized with respect to the parameters to obtain the maximum likelihood estimates. However, the model is overparameterized if separate fishing and natural mortalities are specified for every year. Therefore, it is necessary to specify some restrictions.

To illustrate the approach we consider two models. The first is the most general model in which $F$ and $M$ are allowed to vary from year to year. The second is a standard fishery model in which $M$ is held constant while $F$ varies. Models with various alternative restrictions on the fishing and natural mortality rates are straightforward to implement.

\section{Model with variable $\boldsymbol{F}$ and $\boldsymbol{M}$}

For this model, the fishing and natural mortality rates are allowed to vary from year to year. However, mortality rates can only be estimated for one less than the number of years of tagging (i.e., for $I-1$ years). The estimable parameters are 


$$
\begin{aligned}
& F_{j}, j=1,2, \ldots, I-1 \\
& M_{j}, j=1,2, \ldots, I-1 \\
& f_{I}\left(\text { or } u_{I}\right) \\
& f_{I+1} S_{I} \\
& \cdot \\
& \cdot \\
& \cdot \\
& f_{J} S_{I} S_{I+1} \ldots S_{J-1}
\end{aligned}
$$$$
\phi \lambda
$$

Note that, for year $I$ and beyond, it is not possible to break the exploitation rate $u$ into its component fishing and natural mortality rates; consequently, we drop the notation $u_{j}\left(F_{j}, M_{j}\right)$ in favor of $u_{j}$ to emphasize that the parameter is not further interpretable. The likelihood is given by eq. 2 with the $P_{i j}$ defined by

$$
P_{i j}= \begin{cases}\phi \lambda u_{j}\left(F_{j}, M_{j}\right) & i=j<I \\ \phi \lambda u_{j}\left(F_{j}, M_{j}\right) \prod_{h=i}^{j-1} \mathrm{e}^{-F_{h}-M_{h}} & i<j<I \\ \phi \lambda u_{I} & i=j=I \\ \phi \lambda u_{I} \prod_{h=i}^{j-1} \mathrm{e}^{-F_{h}-M_{h}} & i<j=I \\ \phi \lambda u_{j} S_{I} \ldots S_{j-1} & I=i<j \\ \phi \lambda u_{j} S_{I} \ldots S_{j-1} \prod_{h=i}^{I-1} \mathrm{e}^{-F_{h}-M_{h}} & i<I<j\end{cases}
$$

Note that the product $\phi \lambda$ is estimable in theory if $\phi \lambda$ is constant over time.

\section{Model with variable $F$ and constant $M$}

Natural mortality rate is usually considered constant over time and over recruited ages in fisheries assessments. This is largely due to the difficulty until now of estimating natural mortality. Although in theory one can obtain year-specific estimates of natural mortality, it may be useful to estimate a single value to reduce the mean squared error and for comparative purposes. In this case, the parameters to be estimated are the constant mortality rate, $M$, and the fishing mortality in each recovery year $j, j=1, \ldots, J$. The product $\phi \lambda$ can also be estimated if it is assumed not to vary over time. The likelihood is given by eq. 2 with $P_{i j}$ defined as in Table 2 .

In Pollock et al. (1991), the Brownie models were parameterized in terms of survival, $S$, and tag recovery rate, $f$. Thus, it was possible to estimate a separate $F$ and $M$ for each year (up to year $I-1$ ) or to estimate a single value for $F$ and for $M$ for all years. But, with their framework, it was not possible to impose arbitrary restrictions such as to let $F$ vary from year to year while keeping $M$ fixed.

\section{Assuming catchability is constant from year to year}

There is an extensive literature discussing how fishing effort is related to fishing mortality (see Hutchings and Myers 1994). When the fishing mortality is strongly related to the fishing effort, we can use the effort data to determine the relative amounts of mortality at different times and estimate the parameter (or parameters) necessary to convert from relative mortality to absolute mortality rates.

Assume that the fishing mortality, $F_{j k}$, in period $k$ of year $j$ is proportional to the effort $E_{j k}$ in the period. Then, $F_{j k}=q E_{j k}$, where $q$ is known as the catchability coefficient. $q$ can be thought of as the fraction of the population caught by one unit of fishing effort when that fraction is small (say, less than $1 \%$; Ricker 1975). The expected values of the numbers of recoveries can be derived from those given in Table 2 by replacing $F_{j k}$ by $q E_{j k}$ (Table 3 ). Then in eqs. $5 a$ and $5 b, F_{j}$ is replaced by $q \sum_{k} E_{j k}$.

When the product $\phi \lambda$ is known, the parameters $q$ and $M$ can be estimated from 2 years of recoveries from a single tagged cohort. For example, consider the case of a pulse fishery occurring at the beginning of each year. The expected number of recaptures in the first year is $N_{1} \phi \lambda\left(1-\mathrm{e}^{-q E_{1}}\right)$ and the expected number in the second year is $N_{1} \phi \lambda\left(1-\mathrm{e}^{-q E_{2}}\right) \mathrm{e}^{-q E_{1}-M}$ (Table 3). Setting the observed numbers of recaptures in the first 2 years equal to their expected values provides two equations in two unknowns, which can be solved simultaneously for $M$ and $q$. Estimates of $M$ and $q$ can be obtained in the same way when the effort has an arbitrary distribution over the course of a year. If recoveries are obtained for more than 2 years then it is possible to estimate year-specific natural mortality rates, $M_{j}$, or to estimate more than one catchability coefficient.

If tagging is conducted for 2 years and recoveries are obtained for 2 years, the product $\phi \lambda$ is also estimable. Also, $\phi \lambda$ is estimable if tagging is conducted for 1 year and recaptures are obtained for 3 years. Consider again a pulse fishery occurring at the beginning of each year. We can solve three equations simultaneously for $q, M$ and the product $\phi \lambda$ :

$$
\begin{aligned}
& r_{11}=N_{1} \phi \lambda\left(1-\mathrm{e}^{-q E_{1}}\right) \\
& r_{12}=N_{1} \phi \lambda\left(1-\mathrm{e}^{-q E_{2}}\right) \mathrm{e}^{-q E_{1}-M} \\
& r_{22}=N_{2} \phi \lambda\left(1-\mathrm{e}^{-q E_{2}}\right)
\end{aligned}
$$

provided $E_{1} \neq E_{2}$. Alternatively, we can solve the system

$$
\begin{aligned}
& r_{11}=N_{1} \phi \lambda\left(1-\mathrm{e}^{-q E_{1}}\right) \\
& r_{12}=N_{1} \phi \lambda\left(1-\mathrm{e}^{-q E_{2}}\right) \mathrm{e}^{-q E_{1}-M} \\
& r_{13}=N_{1} \phi \lambda\left(1-\mathrm{e}^{-q E_{3}}\right) \mathrm{e}^{-q E_{1}-q E_{2}-2 M}
\end{aligned}
$$

provided not all fishing efforts are equal.

As before, the example above is easily generalized to allow for an arbitrary pattern of fishing effort over the year. The expressions for exploitation rate and survival rate in Table 3 are replaced with the appropriate modification of eqs. $5 a$ and $5 b$ in which $F_{j}$ is replaced by $q E_{j}$.

It is a straightforward matter to allow $q$ or $M$ or $\phi \lambda$ to vary somewhat from year to year. For example, one might wish to try a model in which $q$ in recent years is higher than in previous years or to explore whether $q$ or $M$ might have changed in years 
Table 2. Instantaneous rates formulation for expected number of tag recoveries in a multiyear tagging study when fishing mortality varies by year and natural mortality is constant over time: (A) all fishing occurs at the beginning of the year; (B) fishing occurs throughout the year with constant intensity; and (C) fishing effort follows an arbitrary pattern over the course of the year.

\begin{tabular}{lccc}
\hline \multicolumn{5}{l}{ (A) Fishing at the beginning of the year. } \\
\cline { 2 - 4 } Year & 1 & 2 & \multicolumn{1}{c}{ Expected recoveries in year } \\
\hline 1 & $N_{1} \phi \lambda\left(1-\mathrm{e}^{-F_{1}}\right)$ & $N_{1} \phi \lambda\left(1-\mathrm{e}^{-F_{2}}\right) \mathrm{e}^{-F_{1}-M}$ & $N_{1} \phi \lambda\left(1-\mathrm{e}^{-F_{3}}\right) \mathrm{e}^{-F_{1}-F_{2}-2 M}$ \\
2 & - & $N_{2} \phi \lambda\left(1-\mathrm{e}^{-F_{2}}\right)$ & $N_{2} \phi \lambda\left(1-\mathrm{e}^{-F_{3}}\right) \mathrm{e}^{-F_{2}-M}$ \\
3 & - & - & $N_{3} \phi \lambda\left(1-\mathrm{e}^{-F_{3}}\right)$ \\
\hline
\end{tabular}

(B) Fishing throughout the year.

\begin{tabular}{lccc}
\hline & \multicolumn{3}{c}{ Expected recoveries in year } \\
\cline { 2 - 4 } Year & 1 & \multicolumn{1}{c}{2} & \multicolumn{1}{c}{3} \\
\hline & $\frac{N_{1} \phi \lambda F_{1}}{F_{1}+M}\left(1-\mathrm{e}^{-F_{1}-M}\right)$ & $\frac{N_{1} \phi \lambda F_{2}}{F_{2}+M}\left(1-\mathrm{e}^{-F_{2}-M}\right) \mathrm{e}^{-F_{1}-M}$ & $\frac{N_{1} \phi \lambda F_{3}}{F_{3}+M}\left(1-\mathrm{e}^{-F_{3}-M}\right) \mathrm{e}^{-F_{1}-F_{2}-2 M}$ \\
3 & - & $\frac{N_{2} \phi \lambda F_{2}}{F_{2}+M}\left(1-\mathrm{e}^{-F_{2}-M}\right)$ & $\frac{N_{2} \phi \lambda F_{3}}{F_{3}+M}\left(1-\mathrm{e}^{-F_{3}-M}\right) \mathrm{e}^{-F_{2}-M}$ \\
& & & $\frac{N_{3} \phi \lambda F_{3}}{F_{3}+M}\left(1-\mathrm{e}^{-F_{3}-M}\right)$ \\
\hline
\end{tabular}

(C) General form.

\begin{tabular}{lcll}
\hline & \multicolumn{3}{c}{ Expected recoveries in year } \\
\cline { 2 - 4 } Year & 1 & \multicolumn{1}{c}{3} \\
\hline 1 & $N_{1} \phi \lambda u_{1}\left(F_{1}, M\right)$ & $N_{1} \phi \lambda u_{2}\left(F_{2}, M\right) \mathrm{e}^{-F_{1}-M}$ & $N_{1} \phi \lambda u_{3}\left(F_{3}, M\right) \mathrm{e}^{-F_{1}-F_{2}-2 M}$ \\
2 & - & $N_{2} \phi \lambda u_{2}\left(F_{2}, M\right)$ & $N_{2} \phi \lambda u_{3}\left(F_{3}, M\right) \mathrm{e}^{-F_{2}-M}$ \\
3 & - & - & $N_{3} \phi \lambda u_{3}\left(F_{3}, M\right)$ \\
\hline
\end{tabular}

Note: Symbols are as follows: $N_{j}$, number tagged and released in year $j ; F_{j}$, instantaneous rate of fishing mortality in year $j ; M$, instantaneous rate of natural mortality; $\phi$, probability of surviving being tagged and retaining the tag (in the short term); $\lambda$, tag reporting rate; $u_{j}\left(F_{j}, M\right)$, exploitation rate in year $j$ as defined by eq. $5 a$. Note that $u_{j}$ is a function of $F_{j}$ and $M$ and depends on the seasonal pattern of fishing.

with unusual environmental conditions. However, one cannot estimate a separate $q$ and $M$ for each year.

Another generalization is to allow for different types of effort, e.g., for gillnets and trawls. Then, instead of assuming $F_{j k}=q E_{j k}$, we have $F_{j k}=q_{1} E_{j k 1}+q_{2} E_{j k 2}$ when there are two types of effort. Here, the additional subscript refers to the type of effort. If the amount of the effort of the various gear types tends to vary in the same way over time, then the estimates of the $q$ s will be highly correlated. In this case, one may need to supply an external estimate of the relative catchability of the gear types, e.g., an estimate of $q_{2} / q_{1}$. Then we would model the fishing mortality as

$$
F_{j k}=q_{1} E_{j k 1}+q_{1}\left(q_{2} / q_{1}\right) E_{j k 2}
$$

and we would estimate just $q_{1}$.

\section{Using catch as a proxy for effort}

It often arises that reliable effort data are not available. The data analyst then has two choices: $(i)$ assume a seasonal pattern for the effort (generally, either constant over the year or concentrated at one point in the year) and check the sensitivity of the results to the assumption, or (ii) use the seasonal distribution of the catch as a substitute for the relative amount of effort at each point in the year. There are two ways that the seasonal distribution of catch can be determined: the total catch in the fishery can be estimated by period of the year through a creel or port sampling survey, or the number of tags recovered in a year (from all cohorts combined) can be tabulated by period of the year and used as a measure of the seasonal distribution of catch. Catch in an interval of time, $C$, can be modelled as a function of the effort, $E$, or fishing mortality, $F$, and the average abundance $(\bar{N})$ during the interval as follows (see Ricker 1975):

$$
C=q E \bar{N}=F \bar{N}
$$

Thus, if the total mortality rate during the year is not too high, the catch in each period of the year will be approximately proportional to the effort. To use catch as a substitute for effort, define the fraction of the catch in year $j$ occurring in period $k$, $\zeta_{j k}$, for $k=1,2, \ldots, K$, to be

$$
\zeta_{j k}=\frac{C_{j k}}{\sum_{h=i}^{K} C_{j h}}
$$

The values of $\zeta_{j k}$ are then substituted for $\varepsilon_{j k}$ in $a_{j k}$ in eq. $5 a$.

A better approximation to the seasonal effort than eq. 6 can be obtained by adjusting the observed catches or tag returns by an approximate survival rate. This can be accomplished using 
Table 3. Expected number of tag recoveries in a multiyear tagging study when fishing mortality is assumed proportional to fishing effort and natural mortality is assumed constant: (A) all fishing occurs at the beginning of the year and (B) fishing occurs throughout the year with constant intensity.

\begin{tabular}{lccc}
\hline \multicolumn{5}{l}{ (A) Fishing at the beginning of the year. } \\
\cline { 2 - 4 } Year & 1 & 2 & \multicolumn{1}{c}{ Expected recoveries in year } \\
\hline 1 & $N_{1} \phi \lambda\left(1-\mathrm{e}^{-q E_{1}}\right)$ & $N_{1} \phi \lambda\left(1-\mathrm{e}^{-q E_{2}}\right) \mathrm{e}^{-q E_{1}-M}$ & $N_{1} \phi \lambda\left(1-\mathrm{e}^{-q E_{3}}\right) \mathrm{e}^{-q E_{1}-q E_{2}-2 M}$ \\
2 & - & $N_{2} \phi \lambda\left(1-\mathrm{e}^{-q E_{2}}\right)$ & $N_{2} \phi \lambda\left(1-\mathrm{e}^{-q E_{3}}\right) \mathrm{e}^{-q E_{2}-M}$ \\
3 & - & - & $N_{3} \phi \lambda\left(1-\mathrm{e}^{-q E_{3}}\right)$ \\
\hline
\end{tabular}

(B) Fishing throughout the year.

\begin{tabular}{lccc}
\hline & \multicolumn{3}{c}{ Expected recoveries in year } \\
\cline { 2 - 4 } 1 & \multicolumn{1}{c}{2} & 3 \\
\hline & $\frac{N_{1} \phi \lambda q E_{1}}{q E_{1}+M}\left(1-\mathrm{e}^{-q E_{1}-M}\right)$ & $\frac{N_{1} \phi \lambda q E_{2}}{q E_{2}+M}\left(1-\mathrm{e}^{-q E_{2}-M}\right) \mathrm{e}^{-q E_{1}-M}$ & $\frac{N_{1} \phi \lambda q E_{3}}{q E_{3}+M}\left(1-\mathrm{e}^{-q E_{3}-M}\right) \mathrm{e}^{-q E_{1}-q E_{2}-2 M}$ \\
3 & - & $\frac{N_{2} \phi \lambda q E_{2}}{q E_{2}+M}\left(1-\mathrm{e}^{-q E_{2}-M}\right)$ & $\frac{N_{2} \phi \lambda q E_{3}}{q E_{3}+M}\left(1-\mathrm{e}^{-q E_{3}-M}\right) \mathrm{e}^{-q E_{2}-M}$ \\
& - & - & $\frac{N_{3} \phi \lambda q E_{3}}{q E_{3}+M}\left(1-\mathrm{e}^{-q E_{3}-M}\right)$
\end{tabular}

Note: Symbols are as follows: $N_{j}$, number tagged and released in year $j ; q$, catchability coefficient; $E_{j}$, fishing effort in year $j ; M$, instantaneous rate of natural mortality; $\phi$, probabiliity of surviving being tagged and retaining the tag (in the short term); $\lambda$, tag reporting rate.

$$
\zeta_{j k^{*}}=\frac{C_{j k} / \hat{S}_{j}^{(k-1) / K}}{\sum_{h=i}^{K} C_{j h} / \hat{S}_{j}^{(h-1) / K}}
$$

in eq. $5 a$ in place of the $\varepsilon_{j k}$; the estimates of $S_{j}$ are obtained by maximizing eq. 2.

Even when nominal effort data are available, it may be better to use catch as the measure of effective effort. This might be the case, for example, if catchability varies substantially over the course of a year.

\section{Example: lake trout in Cayuga Lake}

Youngs and Robson (1975) presented results from a 10-year tagging study of lake trout in Cayuga Lake, New York (see Table 4). Fish were tagged in October with monel jaw tags (W.D. Youngs, personal communication). We assume the retention-survival parameter $\phi$ is 1.0 because the cool water temperature in autumn is not likely to stress the fish and because the jaw tag is durable.

Youngs (1974) assumed the tag reporting rate, $\lambda$, was constant over time and estimated it from the Cayuga Lake data to be $18 \%$. For now, we will assume $\lambda$ is known to be 0.18 .

The timing of the fishery is not given in Youngs' papers. We analyzed the first 3 years of data under five assumptions about the timing to observe how critical it is to determine the timing. We used 3 years of data instead of 10 because 10 years of tagging data is uncommon. The assumptions were (i) fishing took place throughout the year with constant intensity; (ii) all fishing took place at the start of each year; (iii) all fishing took place at the end of the year; (iv) all fishing took place at the midpoint of the year; and ( $v$ ) fishing took place throughout the last 6 months of the year with constant intensity during
Table 4. Tag recovery data for lake trout from Cayuga Lake, New York (from Youngs and Robson 1975).

\begin{tabular}{lrrrrrrrrrrr}
\hline \multirow{2}{*}{$\begin{array}{l}\text { Year } \\
\text { tagged }\end{array}$} & $\begin{array}{c}\text { No. } \\
\text { tagged }\end{array}$ & 1 & 2 & 3 & 4 & 5 & 6 & 7 & 8 & 9 & 10 \\
\hline 1960 & 1048 & 72 & 44 & 8 & 9 & 4 & 4 & 1 & 1 & 1 & 0 \\
1961 & 844 & - & 74 & 30 & 20 & 7 & 4 & 2 & 1 & 0 & 0 \\
1962 & 989 & - & - & 54 & 48 & 13 & 23 & 5 & 4 & 2 & 0 \\
1963 & 971 & - & - & - & 74 & 24 & 16 & 7 & 3 & 1 & 1 \\
1964 & 863 & - & - & - & - & 48 & 40 & 5 & 5 & 2 & 5 \\
1965 & 465 & - & - & - & - & - & 31 & 10 & 6 & 3 & 2 \\
1966 & 845 & - & - & - & - & - & - & 38 & 30 & 6 & 2 \\
1967 & 360 & - & - & - & - & - & - & - & 19 & 6 & 6 \\
1968 & 625 & - & - & - & - & - & - & - & - & 13 & 14 \\
1969 & 760 & - & - & - & - & - & - & - & - & - & 21 \\
\hline
\end{tabular}

this period. Youngs has informed us (personal communication) that the fishing season extended from April through September. No information is available about the temporal pattern of effort within the 6-month fishing season. Thus, it appears that the fifth scenario most closely approximates the actual fishery.

We fitted two models for each assumption about timing. The first model had separate fishing mortalities in each of the 3 years and a constant natural mortality rate over the 3 -year period. The second model had constant fishing and natural mortalities over the 3-year period.

The results are similar for all assumptions about timing of the fishery. The estimates of fishing mortality for year 1 range from $0.50 \cdot$ year $^{-1}$ for a fishery at the beginning of the year to 0.59 for a fishery at the end of the year (Table 5). For the second year, the range of estimates is $0.64-0.74 \cdot$ year $^{-1}$, and for the third year the range is $0.40-0.45$. Thus, under all five 
Table 5. Results of fitting tagging models to 3 years of lake trout recapture data.

\begin{tabular}{|c|c|c|c|c|c|}
\hline \multirow[b]{2}{*}{ Parameter } & \multicolumn{5}{|c|}{ Estimate (SE) under fishery timing assumption } \\
\hline & Continuous & Start of year & End of year & Month 7 & Last 6 months \\
\hline \multicolumn{6}{|c|}{ Model: year-specific $F$, constant $M$} \\
\hline$F_{1}$ & $0.55(0.08)$ & $0.50(0.08)$ & $0.59(0.08)$ & $0.56(0.08)$ & $0.58(0.08)$ \\
\hline$F_{2}$ & $0.70(0.09)$ & $0.64(0.08)$ & $0.74(0.11)$ & $0.70(0.10)$ & $0.72(0.10)$ \\
\hline$F_{3}$ & $0.43(0.08)$ & $0.40(0.06)$ & $0.45(0.10)$ & $0.43(0.08)$ & $0.44(0.09)$ \\
\hline$M$ & $0.16(0.09)$ & $0.22(0.11)$ & $0.11(0.06)$ & $0.15(0.08)$ & $0.13(0.07)$ \\
\hline $\operatorname{Corr}\left(F_{1}, M\right)$ & -0.08 & -0.38 & 0.15 & -0.05 & 0.05 \\
\hline $\operatorname{Corr}\left(F_{2}, M\right)$ & 0.43 & 0.12 & 0.59 & 0.47 & 0.53 \\
\hline $\operatorname{Corr}\left(F_{3}, M\right)$ & 0.67 & 0.47 & 0.75 & 0.69 & 0.72 \\
\hline \multicolumn{6}{|c|}{ Model: constant $F$, constant $M$} \\
\hline$F$ & $0.58(0.06)$ & $0.51(0.04)$ & $0.64(0.07)$ & $0.59(0.06)$ & $0.61(0.07)$ \\
\hline$M$ & $0.23(0.07)$ & $0.30(0.09)$ & $0.17(0.05)$ & $0.22(0.07)$ & $0.19(0.06)$ \\
\hline $\operatorname{Corr}(F, M)$ & 0.44 & 0.11 & 0.56 & 0.45 & 0.52 \\
\hline
\end{tabular}

assumptions about timing we would come to the same qualitative conclusion that the trout population is heavily exploited. Similarly, the estimates of natural mortality under these models range from $0.11 \cdot$ year $^{-1}$ for a fishery at the end of the year to 0.22 for a fishery at the beginning of the year. As previously noted, the most appropriate assumption about timing of fishing appears to be that of uniformity over the last 6 months of the year. The estimates under this assumption (last column) are close to the estimates for all other assumptions except perhaps the pulse fishery at the start of the year (second column).

For the more restricted models with fishing mortality constant over time, the estimates of $F$ range from 0.51 to 0.64 and the estimates of $M$ range from 0.30 to 0.17 (Table 5). Again, except for the model with all fishing occurring at the start of the year, the estimates are in close agreement.

\section{Further sensitivity analyses and simulations}

We fitted to the 10-year lake trout data set a model in which fishing mortality was allowed to vary from year to year, and natural mortality and tag reporting rate were unknown but constant over time. The retention-survival parameter $\phi$ was assumed to be 1.0, and fishing effort was assumed to be distributed uniformly over the year. Following Youngs (1974) we assumed that fishing occurred with constant intensity over the entire year. Our estimate, $\hat{\lambda}=0.19$, was close to the estimate that Youngs obtained from the same data using an alternative, less efficient estimator. We then asked how reliable (stable) is this estimate. In particular, we wished to determine if reasonable estimates could be obtained with fewer than 10 years of data. We refitted the model using the first 9 years of data and also using the last 9 years of data. We then fitted the model using 8 years of data (i.e., years $1-8,2-9$, and $3-10)$. Continuing in this fashion, we found that the estimates are highly unstable (Fig. 1). Even with 9 years of data, the two estimates were far apart. With less than 9 years of data we frequently obtained estimates of natural mortality equal to zero. With 3 years of data the computations failed to converge twice. Therefore, it appears that the information on tag reporting rate in the data is extremely weak and additional kinds of information are required to obtain reliable estimates of reporting rate.

The computer program failed to converge when we attempted to estimate the fishing mortality rates in the first 3 years and the natural mortality rate and tag reporting rate from 3 years of recapture data from the first three tagged cohorts. We then investigated the impact on the analysis of having data from a study with high-reward tags. (We asssume the reward is so high that reporting rate for these tags is $100 \%$.) We simulated the return of high-reward tags for the cases where $1,2,5$, or $10 \%$ additional tags were released each year with high rewards. To generate these data, we assumed that the fishing mortalities and natural mortality in the first 3 years were equal to the estimates obtained from the full 10-year data set. We generated 500 sets of simulated tag returns for each level of additional tags.

In all cases, the computations converged though not necessarily to feasible estimates. When $1 \%$ additional tags were released with high rewards, the estimates of natural mortality rate were sometimes negative and were extremely variable (range -0.26 to 0.52 ) (Fig. 2). With $2 \%$ additional tags, reporting rate ranged from 0.14 to 0.32 , while the estimates of natural mortality ranged from -0.06 to 0.43 year $^{-1}$. With $5 \%$ additional tags, the range of the estimates of reporting rate shrank to $0.16-0.27$; the range of the natural mortality estimates was $0.02-0.36$. With $10 \%$ additional tags, the estimates of both reporting rate and natural mortality were somewhat more precise (range for reporting rate $0.16-0.25$; range for $M 0.07-$ $0.31)$.

Releasing $2 \%$ more tags meant that 21,17 , and 20 tags were released in the 3 years. If the tags carried a reward of $\$ 100$, say, and the exploitation rate was roughly $35 \%$, then the additional cost in terms of rewards would be about $\$ 3000$ total over a 3 -year period. Similar calculations for the case where $5 \%$ additional tags were released implies an added cost of rewards of $\$ 7500$. Thus, it appears that the high-reward study is effective, and the cost need not be prohibitive. 
Fig. 1. Estimates of tag reporting rate, $\lambda$, (upper panel) and instantaneous natural mortality rate, $M$, (lower panel) for lake trout in Cayuga Lake. The abscissa gives the number of years of tagging and recovery data used. Abscissa values have been jittered (a small random number has been added to each value) to improve the readability. There are two ways to divide the 10-year data set into continuous 9 -year blocks (i.e., using years 1-9 or 2-10), three ways to divide the data into 8-year blocks, etc. Note that, even with 9 years of data, the estimates are highly variable. The computer algorithm failed to converge twice when only 3 years of tagging and recovery data were considered.
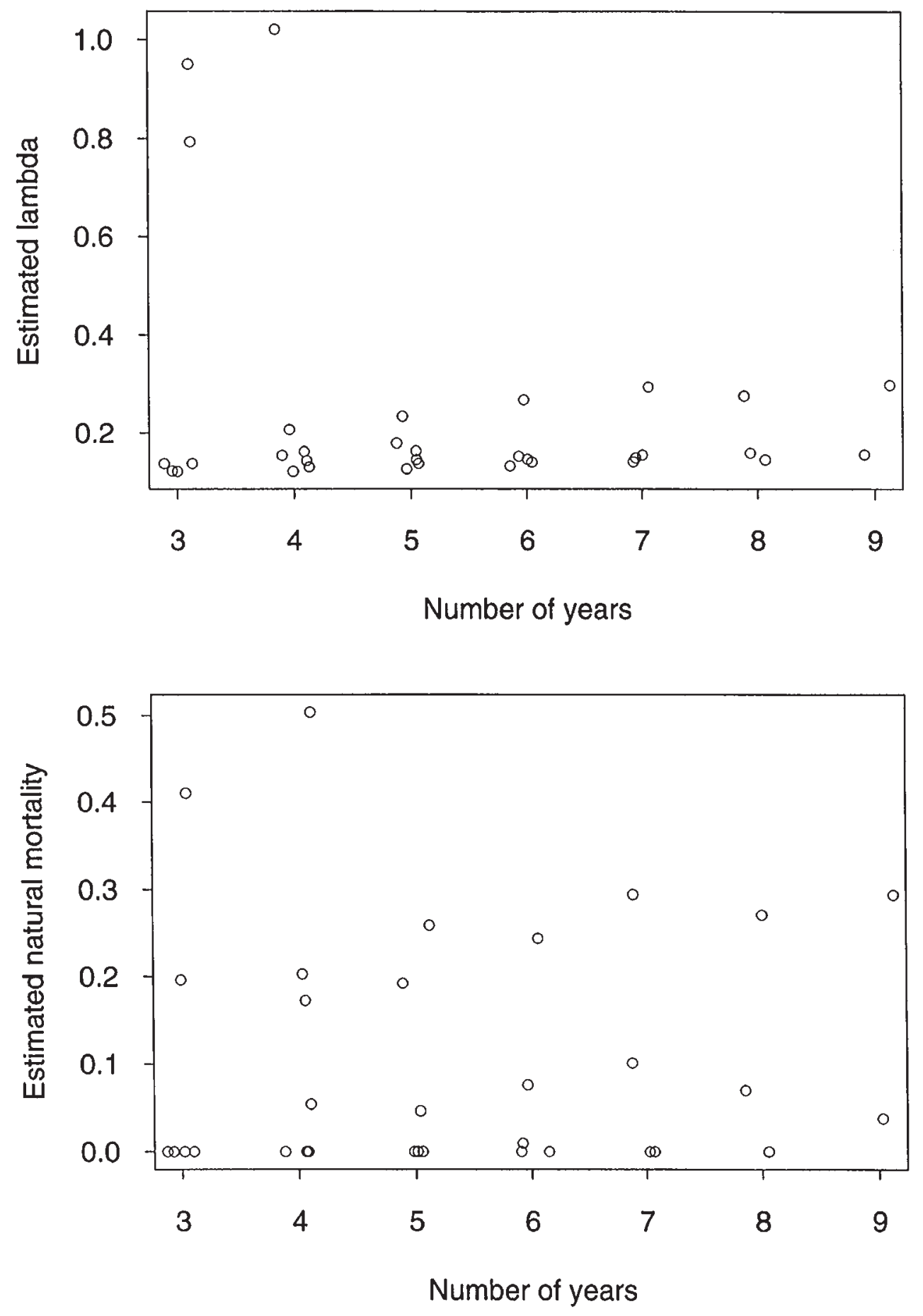

\section{Discussion}

\section{Weak assumption about effort}

We have shown that information on timing of the fishery can be used to convert estimates of exploitation and survival rates into estimates of fishing and natural mortality. Fortunately, in our example it appears that estimates of mortality rates are not overly sensitive to misspecification of the timing so that even crude timing information may suffice. Because the effort data can be crude, it is even possible to use the seasonal distribution of catch (or tag recaptures) as a proxy for effort data.

The instantaneous rates formulation of the tagging models 
Fig. 2. Estimates of tag reporting rate (left) and natural mortality rate (right) from a 3-year tagging study (lake trout data 1960-1962) with addition of simulated returns from an additional 1\% (top), $2 \%, 5 \%$, or 10\% (bottom) tags released each year with high rewards. Without the simulated high-reward tags, the computations failed to converge.
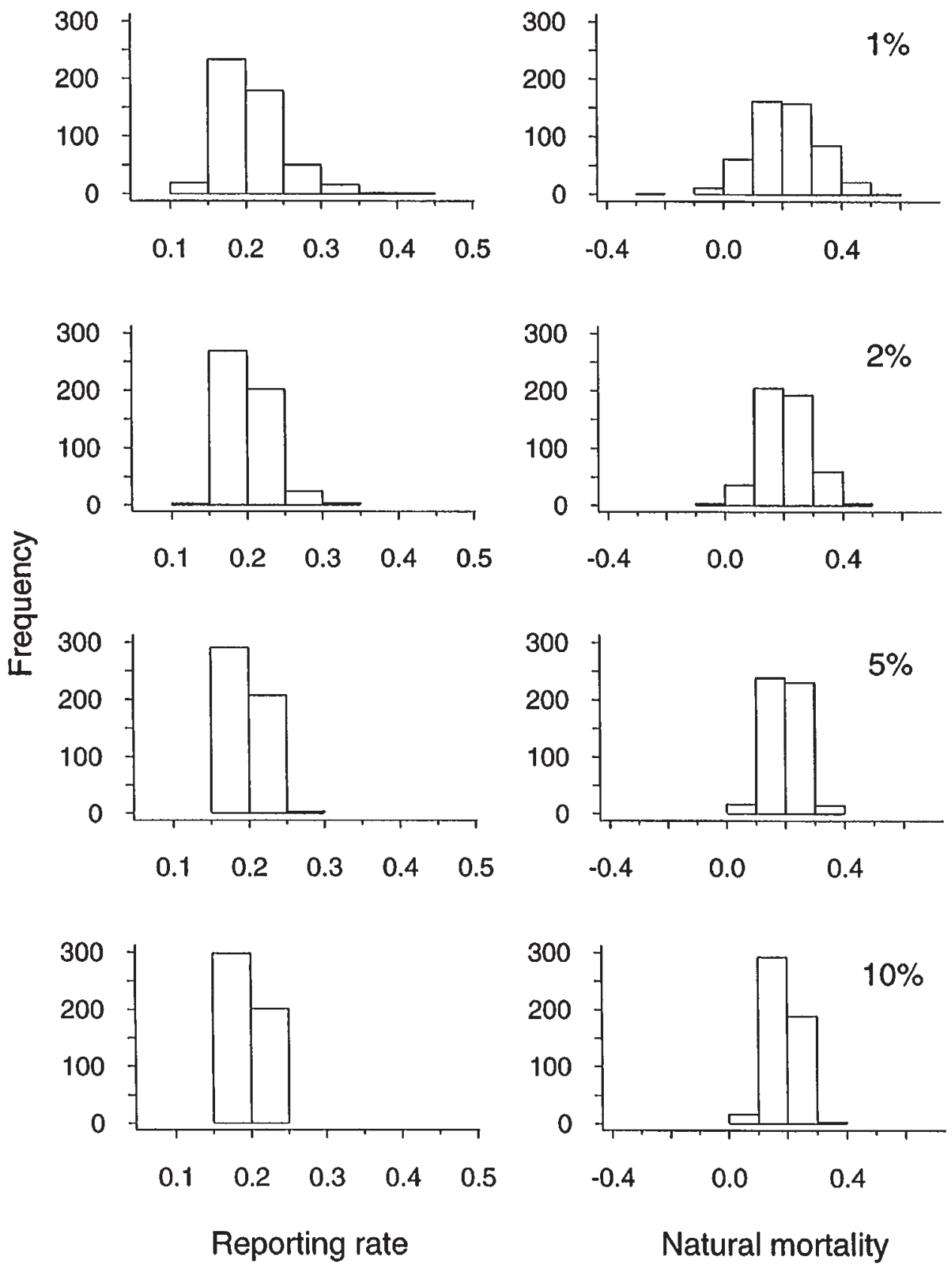

is important, because it allows meaningful restrictions to be imposed on the parameters such as constant natural mortality rates, separate natural mortality rates for cold and for normal years, or fishing mortality proportional to fishing effort. Formulating the tagging models in terms of instantaneous rates also bridges the gap between traditional fishery models and models of tagging data developed in the wildlife and ecological literatures. With further work, it may be possible to generalize Brownie-like tagging models to incorporate an index of abundance and the catch at age matrix.

It is clear that, in theory, the product $\phi \lambda$ can be estimated from tagging data alone (Brownie 1974; Youngs 1974).
However, it appears that, in practice, it will generally be necessary to obtain additional information on tag reporting rate from a variable-reward tagging study, planted tags, or a creel or plant sampling survey (see Appendix). Our estimates of the lake trout reporting rate were not stable without additional information and Brownie's (1974) attempt to apply a version of the model to mallard duck (Anas platyrhynchos) data was also unsatisfactory. Fortunately, it appears that even modest numbers of tag returns with high rewards are enough to stabilize the estimate of $\lambda$ (assuming $\phi$ is known) and thus to stabilize the estimate of $M$.

It is interesting that the estimates of $F$ and $M$ are only 
moderately correlated in the trout example and in our simulations. This suggests that it might be practical to obtain reliable estimates of natural mortality for many stocks if the study is designed carefully (see Pollock et al. 1991 for a review of the necessary assumptions). In particular, it is necessary to tag fish over the entire geographic range of the stock to ensure that the tagged population is representative of the target population, to estimate the tag-shedding and tag-induced mortality rates with cage studies, and to include a method of estimating tag reporting rate in the study.

Youngs (1974) estimated the tag reporting rate, $\lambda$, for anglers catching lake trout on Cayuga Lake from the full 10-year data set. He began by fitting his early version of what has come to be known as a Brownie model and thus estimated the annual survival rates. He then regressed the instantaneous total mortality rate $Z_{i}\left(Z_{i}=-\ln \left(S_{i}\right)\right)$ against the tag recovery rate parameter $f_{i}$ and took the reciprocal of the slope to be an estimate of the tag reporting rate. His analysis is not internally consistent, because he allowed $S$ and $f$ to vary freely from year to year in the first part of his analysis; however, in the second part of the analysis he assumed that $\lambda$ and $M$ were constant, and this would imply that $f$ is a one-to-one function of $S$. Even though his estimator is not fully efficient, his work is notable because he clearly demonstrated that there is information about the tag reporting rate in the tagging data and he pointed the way towards parameterizing the tagging models in terms of instantaneous rates.

\section{Strong assumption about effort}

There is a large literature critical of the assumption that fishing mortality is directly proportional to fishing effort. Nonetheless, developing models based on this assumption is attractive because many fewer parameters need to be estimated. This can result in substantial improvements in precision even if some bias is introduced because of model misspecification. When the recovery matrix is sparse, it may be necessary to invoke the strong assumption about effort to make sense of the data.

Lucas (1975) developed a model for a single release of tagged animals that makes use of the strong effort assumption. He modelled the exact times of recaptures rather than the number of recaptures per interval of time. He also derived an approximate expression for the probability that an animal is not recaptured during the study. A simpler, exact expression is available, however. The principal difference between Lucas' and our approach is that Lucas implicitly assumed the tag reporting rate was 1.0 , whereas we show that reporting rate can either be estimated from the tagging data, specified explicitly in the analysis, or estimated from the joint analysis of tagging and other data. It was apparently previously unrecognized that reporting rate could be estimated from a single-release tagging experiment.

\section{Acknowledgements}

We thank Dennis Heisey for calling relevant literature to our attention, Cavell Brownie for supplying us with her unpublished manuscript on instantaneous rates formulation, and two anonymous reviewers who provided helpful comments.

\section{References}

Barrowman, N.J., and Myers, R.A. 1996. Estimating tag shedding rates for experiments with multiple tag types. Biometrics, 52: 1410-1416.

Brownie, C. 1974. Estimation of instantaneous mortality rates from band return data when "within-season date of kill" is also available. Biometrics Unit Mimeo Ser. Pap. No. BU-536-M Cornell University, Ithaca, N.Y.

Brownie, C., Anderson, D.R., Burnham, K.P., and Robson, D.S. 1978. Statistical inference from band recovery data—a handbook. U.S. Fish Wildl. Serv. Resour. Publ. No. 131.

Brownie, C., Anderson, D.R., Burnham, K.P., and Robson, D.S. 1985. Statistical inference from band recovery data: a handbook. 2nd ed. U.S. Fish Wildl. Serv. Resour. Publ. No. 156.

Conroy, M.J. 1985. Maximum likelihood methods for investigating reporting rates of rings of hunter-shot birds. In Statistics in ornithology. Edited by B.J.T. Morgan and P.M. North. SpringerVerlag, New York. pp. 215-241.

Conroy, M.J., Hines, J.E., and Williams, B.K. 1989. Procedures for the analysis of band-recovery data and user instructions for program MULT. U.S. Fish. Wildl. Serv. Resour. Publ. No. 175.

Costello, T.J., and Allen, D.M. 1968. Mortality rates in populations of pink shrimp, Penaeus duorarum, on the Sanibel and Tortugas grounds, Florida. Fish. Bull. U.S. 66: 491-502.

Dorazio, R.M. 1992. Pre-release stratification in tag recovery models with time dependence. Can. J. Fish. Aquat. Sci. 50: 535-541.

Green, A.W., Matlock, G.C., and Weaver, J.E. 1983. A method for directly estimating the tag-reporting rate of anglers. Trans. Am. Fish. Soc. 112: 412-415.

Hearn, W.S., Leigh, G.M., and Beverton, R.J.H. 1991. An examination of a tag-shedding assumption, with application to southern bluefin tuna. ICES J. Mar. Sci. 48: 41-51.

Hearn, W.S., Brooks, E., and Pollock, K.H. 1998. Pre- and postseason tagging models: estimation of reporting rate and fishing and natural mortality rates. Can. J. Fish. Aquat. Sci. 55: 199-205.

Henny, C.J., and Burnham, K.P. 1976. A reward band study of mallards to estimate band reporting rates. J. Wildl. Manage. 40: 1-14.

Hutchings, J.A., and Myers, R.A. 1994. What can be learned from the collapse of a renewable resource? Atlantic cod, Gadus morhua, off Newfoundland and Labrador. Can. J. Fish. Aquat. Sci. 51: 21262146.

Jagielo, T. 1991. Synthesis of mark-recapture and fishery data to estimate open-population parameters. Am. Fish. Soc. Symp. 12: 492-506.

Larson, S.C., Saul, B., and Schleiger, S. 1991. Exploitation and survival of black crappies in three Georgia reservoirs. N. Am. J. Fish. Manage. 11: 604-613.

Lucas, C. 1975. A method for estimating mortality rates from tag recoveries when fishing is not constant. Aust. J. Mar. Freshwater Res. 26: 75-79.

Pollock, K.H., Hoenig, J.M., and Jones, C.M. 1991. Estimation of fishing and natural mortality when a tagging study is combined with a creel survey or port sampling. Am. Fish. Soc. Symp. 12: 423-434.

Pollock, K.H., Tsai, K., and Hoenig, J.M. 1994. Band return models: a generalization to incorporate solicited bands and allow separation of hunting and natural mortality. J. Wildl. Manage. 58: 193-198.

Pollock, K.H., Conroy, M.J., and Hearn, W.S. 1995. Separation of hunting and natural mortality using ring-return models: an overview. J. Appl. Stat. 22: 557-566.

Ricker, W.E. 1975. Computation and interpretation of biological statistics of fish populations. Bull. Fish. Res. Board Can. No. 191.

Robson, D.S. 1971. Statistical analysis of reported tag-recaptures from an exploited population: maximum likelihood estimation of 
instantaneous mortality rates. Biometrics Unit Mimeo Ser. Pap. No. BU-370-M. Cornell University, Ithaca, N.Y.

Robson, D.S., and Youngs, W.D. 1971. Statistical analysis of reported tag recaptures in the harvest from an exploited population. Biometrics Unit Mimeo Ser. Pap. No. BU-369-M. Cornell University, Ithaca, N.Y.

Schwarz, C.J., Schweigert, J.F., and Arnason, A.N. 1993. Estimating migration rates using tag recovery data. Biometrics, 49: 177-193.
Seber, G.A.F. 1970. Estimating time-specific survival and reporting rates for adult birds from band returns. Biometrika, 57: 313-318.

Youngs, W.D. 1974. Estimation of the fraction of anglers returning tags. Trans. Am. Fish. Soc. 103: 616-618.

Youngs, W.D., and Robson, D.S. 1975. Estimating survival rates from tag returns: model tests and sample size determination. J. Fish. Res. Board Can. 32: 2365-2371.

\section{Appendix. Incorporating information on tag reporting rate.}

There are three basic ways to obtain information on tag reporting rate: (i) planted tags, (ii) variable (high) reward studies, and (iii) catch (creel or port) sampling surveys. These have been reviewed by Pollock et al. (1995).

In a planted tag study, tagged animals are placed in the catch of randomly selected fishers, and the fraction of the tags returned is taken as an estimate of the reporting rate. If each decision by a fisher on whether or not to return a tag (any tag) is made independently of the decisions for other tags then the number of planted tags reported can be taken to be a binomial random variable with parameter equal to the reporting rate in the fishery as a whole. This is closely approximated when the expected number of tag recoveries by a fisher is small (say, less than one). However, the procedure is ill defined if fishers tend not to bother returning a single tag (say, because the reward is small) but send in tags when several have accumulated.

On the assumption that returns of planted tags follow a binomial distribution, then if $x$ tags are returned out of $n$ planted, the likelihood for the planted tag data is

(A1) $\Lambda_{p}=\left(\begin{array}{l}n \\ x\end{array}\right) \lambda^{x}(1-\lambda)^{n-x}$

where $\lambda$ is the tag reporting rate. The likelihood (eq. 2) for regular tags is multiplied by the likelihood (eq. A1) for planted tags to get the joint likelihood for both types of data.

In a high reward tag study, a number of tagged animals are released with messages that clearly indicate the tags can be returned for a high reward. A publicity campaign is conducted so that the fishing community is aware of these high-reward tags. The assumption is made that the rewards are sufficiently high and the publicity campaign is sufficiently extensive that the tags from $100 \%$ of the recaptured animals are reported. The reporting rate for standard tags can be estimated as the ratio of the proportion of returns of standard to high-reward tags.

The expected value of the number of returns from the $i$ th release of high-reward tags captured in year $j$ is exactly the same as for standard tags (e.g., in Table 2) except that everywhere that $\lambda$ occurs it is replaced by 1.0 . Thus, the likelihood for the high reward tag data is constructed in exactly the same way as for the standard-tag data (except for the $\lambda$ ) and the joint likelihood for both types of data is the product of the two likelihoods.

When a port sampling or creel survey is conducted, there are two types of tag returns: those that are solicited directly by the survey agent from the fishers and those that are voluntarily returned by fishers who have not encountered a survey agent. The former are assumed to be surrendered or reported with probability 1.0 , while the latter are reported with probability $\lambda$. If $\delta$ is the fraction of the fishery that is surveyed (e.g., $\delta=$ 0.10 if $20 \%$ of the access points are surveyed on $50 \%$ of the days of the fishing season) then $100 \delta \%$ of the tags from recaptured fish are expected to be recovered from the survey, leaving $100(1-\delta) \%$ of the tags to be voluntarily reported with probability $\lambda$. Thus, we again have two sets of expected values. For solicited tags, the expected number of returns from year $j$ $(j \geq i)$ is

$$
E_{s}\left(R_{i j}\right)=N_{i} f_{j s} \prod_{h=i}^{j-1} S_{h}=N_{i} u_{j} \delta_{j} \prod_{h=i}^{j-1} S_{h}
$$

while for tags reported without solicitation the expected value is

$$
E_{r}\left(R_{i j}\right)=N_{i} f_{j r} \prod_{h=i}^{j-1} S_{h}=N_{i} u_{j}\left(1-\delta_{j}\right) \prod_{h=i}^{j-1} S_{h}
$$

where $\Pi S_{h}$ is defined to be 1.0 if $j=i$. The tags recovered by solicitation and by voluntary reporting (as well as those never recovered) together form one multinomial distribution for each year of tagging. 